\title{
Surface Clutter Reduction for Ground Penetrating Radar
}

\author{
Queen Hesti Ramadhamy ${ }^{1}$, Erfansyah $\mathrm{Ali}^{2}$, A. A. Pramudita ${ }^{3}$ \\ ${ }^{12}{ }^{3}$ School of Electrical Engineering, Telkom University \\ ${ }^{1}$ queenhestir@student.telkomuniversity.ac.id \\ 2erfansyahali@telkomuniversity.ac.id \\ ${ }^{3}$ pramuditaadya@telkomuniversity.ac.id
}

Manuscript received September 12, 2021; revised November 10, 2021; accepted December 23, 2021

\begin{abstract}
Ground Penetrating Radar (GPR) utilizes electromagnetic waves transmitted to the ground and serve as a non-destructive technique. In the detection process, electromagnetic waves touch the surface clutter and to underground to detect the buried object. Ground reflected signal, called surface clutter, is received along with object reflected signal unexpected. Surface clutter is one of the problem in GPR survey that may caused the difficulty in detecting object. Clutter reduction can improve detection results. Averaging method is one of the methods to improve the detection result. In this experiment, GPR system is modelled using Vector Network Analyzer (VNA) and the averaging method is applied on object object detection which is recognized more clearly. The result obtained after the surface clutter reduction process at $3 \mathrm{~cm}$ depth is the disruption of the hyperbolic shape from the object due to its position adjacent to the surface clutter. Whereas at $10 \mathrm{~cm}$ depth, the hyperbolic signal from the object is not disturbed due to its position far from the surface clutter.
\end{abstract}

Keywords: GPR, surface clutter, VNA, averaging method

DOI: $10.25124 /$ jmecs.v8i2.2869

\section{Introduction}

Lots of activities require information about conditions below the ground surface such as military, civil engineering, and geophysics. In this era, engineers developed a Ground Penetrating Radar (GPR) to complete those activities with a nondestructive method for ground surface. Several functions of GPR are including pipes and detect land mines, concrete thickness measurement, detect void, and evaluate the corrosion $[1,12,15]$. GPR utilizes the principle of scattering of electromagnetic waves to determine the location of a buried object by emits those waves to the ground and receives the echo signals [3]. These echoes are processed and served as detections in form of A-scan or B-scan. A-scan detection is in one dimensional meanwhile, B-scan is in two dimensional [4]. The GPR is modelled by VNA in the experiment. The obtained signal from VNA is a hyperbolic pattern, which is a superposition of coupling signal, surface clutter signal, and the object signal.

In signal processing, the clutter made the detection performance of GPR limited [5]. Besides, another problem is the backscattering signal from the object, which makes the object that is close to the surface difficult to be detected [6]. One of method for reducing the clutter is average method [13]. This method is a simple method that can increase the accuracy estimation $[7,8]$. According to research [12], the average method divided into three, namely realization, angular, and frequency averaging. In realization averaging, the average ensemble obtained from heterogeneous soil surface samples with the same statics. In angular averaging defined as a change in the angle of incidence and angle of scattering. In frequency averaging, it used to simulate the scattering of buried objects 
placed under a heterogeneous soil surface.

This study focuses on processing GPR data obtained from the VNA experiment. The experiments are conducted by detecting signal from the buried cans at $3 \mathrm{~cm}$ and $10 \mathrm{~cm}$ underground which are further called A and scenario B, respectively. In order to avoid problem in detection, clutter reduction was carried out using the averaging method. In this study, the obtained A-scan data is collected into B-scan to determine the presence of clutter, then the range clutter is taken for each A-scan and calculated into Eq. 5. In the final stage of this study, the results of the B-scan in hyperbolic pattern from the object are clearer when the clutter is reduced.

\section{Surface Clutter Reduction Method}

GPR divided into transmitter block and receiver block. In the transmitter block, there are transmitter antenna and pulse generators. On another side, a receiver block consists of a receiver antenna and a central unit for displaying the final data after post signal processing. Figure 1 explains the working principle of GPR, where the transmitter block, which is pulse generator will produce an electrical pulse with the form of pulse repetition frequency $(p r f)$, energy, and a certain duration. This pulse transmitted by the transmitter antenna to detect buried objects under the ground surface. In most practical situations, to apply the constant speed technique used to estimate the depth of an object, an estimate can be made based on constants from the ground that correspond to the speed calculated using Eq. (1). The depth of a object $(h)$ can be shown in Eq. (2). The reflected result from the electromagnetic wave symbolized by $S_{1}, S_{2}$ and $S_{3}$ which are represented the reflected signal between two antennas, reflected from ground surface and reflected from the object, respectively [12].

$$
v=\frac{c}{\sqrt{\epsilon}}
$$

where $c$ is speed of light and $\epsilon$ is relative permittivity.

$$
h=\frac{1}{2} v t
$$

where $v$ is constant velocity and $t$ is transit time. Referring to the Figure 1, the receive signal $\left(S_{r}\right)$ can be written as

$$
S_{r}(t)=S_{1}(t)+S_{2}(t)+S_{3}(t)
$$

where $S_{1}$ is coupling antenna signal directly from antenna to antenna, $S_{2}$ is the reflected signal from the surface and $S_{3}$ is the reflected signal from the surface and the buried object.

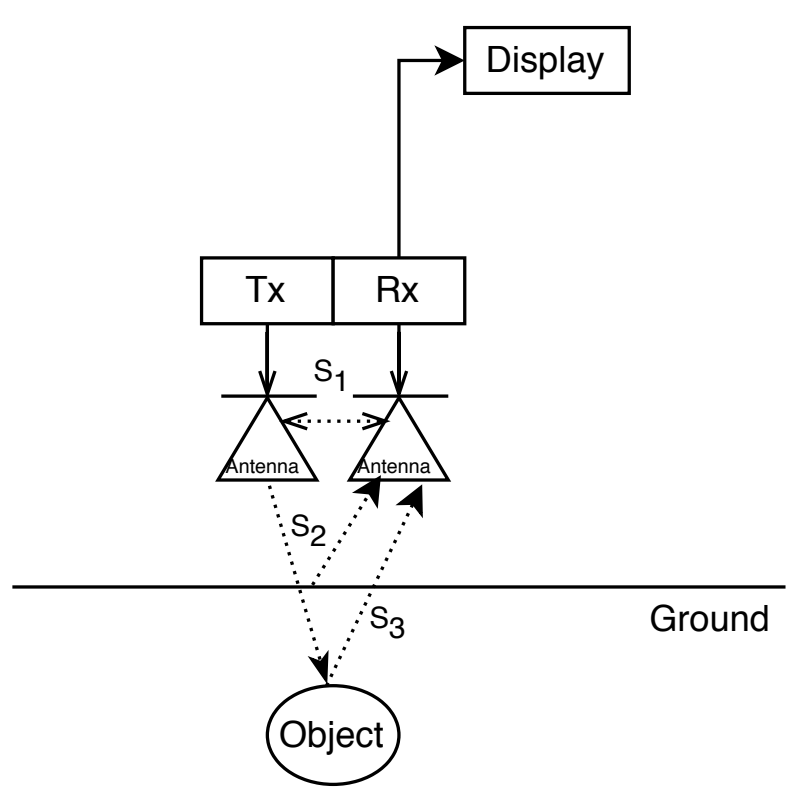

Fig. 1. Illustration of GPR work

This pulse experience attenuation and other signal defects during the propagation on the ground to detect the buried object. The pulse touches the ground surface first, then proceeds to hit the object and reflected into the receiver antenna $(\mathrm{Rx})$. If the ground is homogeneous, the reflected signal clutter is small. If the pulse hits a heterogeneous ground, the reflected signal clutter is bigger than homogeneous. The received signal processed using the software in the central unit and rendered as an image which divided into A-scan, B-scan and C-scan $[1,14]$.

A-scan signal is the presentation of one dimensional data obtained from stationary measurements and signal collection at the desired position. In Figure 2 there are two axes, namely horizontal axes $(x)$ as the surface of the plane and vertical axes $(t)$ as the alternating time of electromagnetic waves. The signal transmitted from antennas to the ground surface has time for the signal to reach the bottom of the surface ground to find the whereabouts of buried objects. Each antennas shift has a different receiving time and waveform due to the shape of the layer of the ground detected and how close the signal transmission to the object detected. The main value of A-scan is monitoring data quality control.

$$
S_{A}=S_{r}(t, x)
$$

where $S_{r}$ is the point from which data was taken, $t$ is alternating time of electromagnetic waves, and $x$ is the surface ground as in (3). 


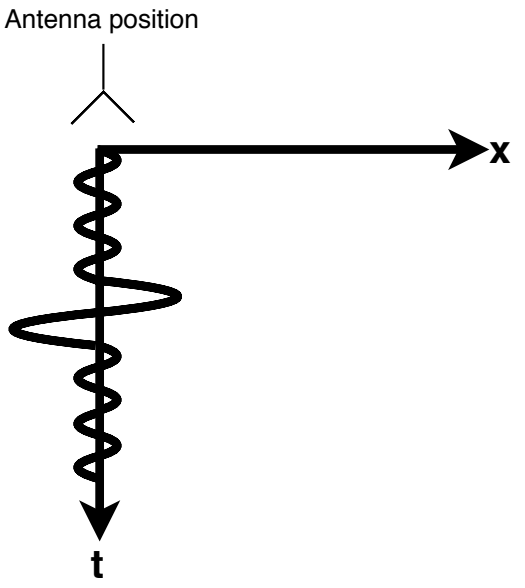

Fig. 2. Presentation of A-scan

On A-scan processing, the form of the received time wave is obtained from the convolution of some time which functions to represent the impulse response of the radar component of the system in addition to noise, then it is accepted in the form of time waves. The impulse response object composed of the convolution from the desired object with other reflections. Some methods that applied in A-scan are zero offset removal, noise reduction, clutter reduction, time-varying gain, frequency filtering, and wavelet optimization or deconvolution techniques [1]. The method in this letter reduces the surface clutter by the averaging method given by Eq. (5).

$$
S_{A}=\frac{1}{n} \sum_{a=1}^{n} S_{2}
$$

where $n$ is number of clutter and $S_{2}$ is surface clutter.

This method works well for a limited number of objects and is physically separated. The sum of the average values includes contributions from all objects. The greater the number, the less difference is produced. The situation that occurs at the planar interface in the area of interest, has the effect of removing part of the wavelength [1].

B-scan signal is a presentation of two dimensional data obtained from the collection of A-scan on the horizontal axis. Another term for twodimensional data is radar profile or radargram [11]. The horizontal axis in Figure 3 is the surface position and the vertical axis is the alternating time of electromagnetic waves. The B-scan used to assure the depth of the object under the ground [1].

Data collection for detecting buried objects using GPR modeled by VNA shown in Figure 4. VNA sends electromagnetic waves to the object

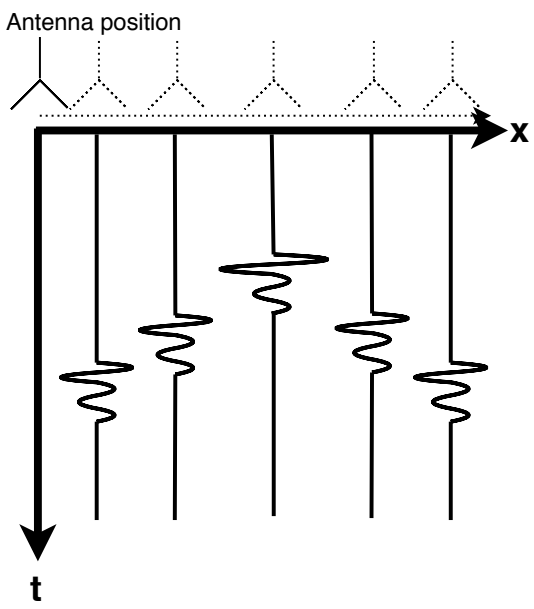

Fig. 3. B-scan signal

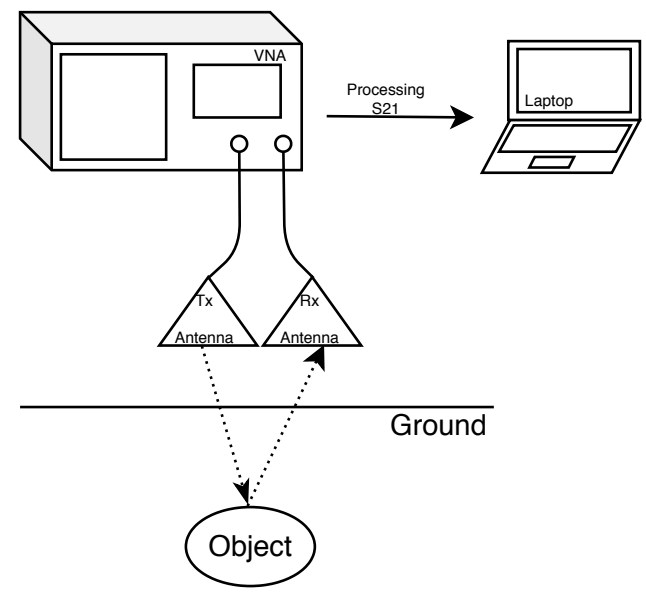

Fig. 4. Modelling using VNA

through the transmitter antenna $(\mathrm{Tx})$ and received by receiver antenna $(\mathrm{Rx})$, which displayed on the VNA monitor. In this experiment, the scattering parameter used is $S_{21}$, where port 1 and port 2 respectively used for the transmitter antenna and the receiver antenna. Collected data by VNA arranged in polar form with real imaginary mode $(R+I m)$ and stored as a CSV file format [10]. The formula used to represent VNA when retrieving $S_{21}$ data shown in Eq. (6).

$$
H(f)=\frac{S_{r}(f)}{S_{t}(f)}
$$

where $H(f)$ is represent $S_{21}, S_{t}(f)$ is transmitted signal, and $S_{r}(f)$ is received signal. The $S_{21}$ data received from the GPR signal can be recruited using the IFFT relationship written as Eq. (7).

$$
S_{r}(t)=F^{-1}\left[S_{21}(f) S_{i}(f)\right]
$$

where $S_{i}$ is the fourier transform of the monocycle signal as a waveform test. The monocycle signal 
is written as Eq. (8).

$$
S_{i}(t)=\frac{1}{\sigma \sqrt{2 \pi}}(t-\tau) \exp \frac{(t-\tau)^{2}}{2 \sigma^{2}}
$$

where $t$ is scaling parameter that sets the pulse width and $\mathrm{s}$ is shift parameter for pulse location.

\section{Design of Experiment}

Data collected in the sandbox that placed outside the room. The experiment setup is shown in Figure 5. The sandbox is constructed from wood and has no impact on the measurement result. Meanwhile, the sand is heterogeneous. The size of sandbox used is $1 \times 1 \times 1 \mathrm{~m}$ and $t_{p}$ is $50 \mathrm{~cm}$. The used antenna type is Vivaldi with a range frequency of 1-10 GHz, where the distance between the antennas $(d)$ is $8 \mathrm{~cm}$. The distance between the antenna to the ground surface is $12 \mathrm{~cm}$. The range frequency of VNA is $0.3-8000 \mathrm{MHz}$.

In this experiment, the object used is a cylindrical and conductive can. It has dimensions of 5 $\mathrm{cm}$ and $13 \mathrm{~cm}$ in diameter and height, respectively. This experiment has two scenarios, which are sceA-4 td.png nario A where the object buried $3 \mathrm{~cm}$ under the surface and scenario B where the object buried $10 \mathrm{~cm}$ under the surface.

The A-scan data obtained by scanning one point to got the signal and then scanning it in another point to get the B-scan data. Data processing undergoes several processes, namely A-scan and B-scan. The process is carried out sequentially.

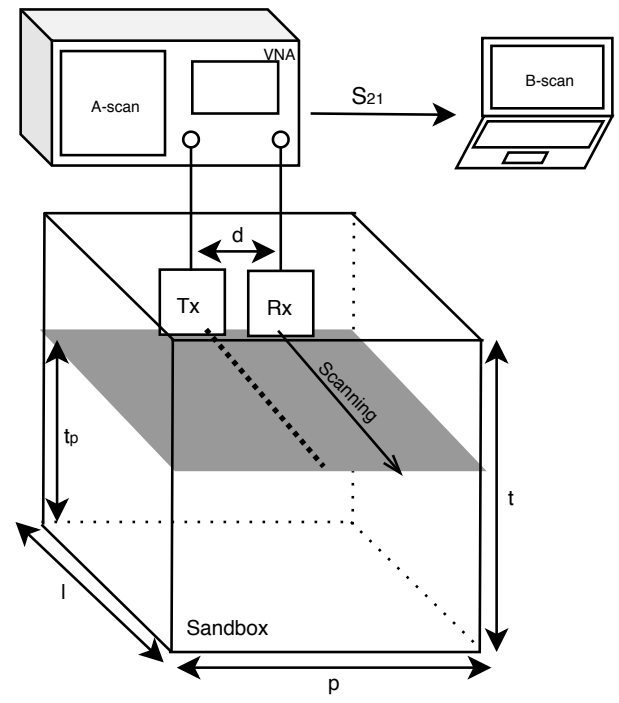

Fig. 5. Experiment design

\section{Result and Discussion}

The reduction of surface clutter can be if seen from the quality of the reflected signal B-scan quantitatively and qualitatively. Qualitatively, reducing surface clutter can affect the level of clarity of reflected objects in the form of hyperbolic pattern where the surface clutter reduced B-scan show clearer results than original B-scan. The quantitatively is to view the accuracy of the object's position.

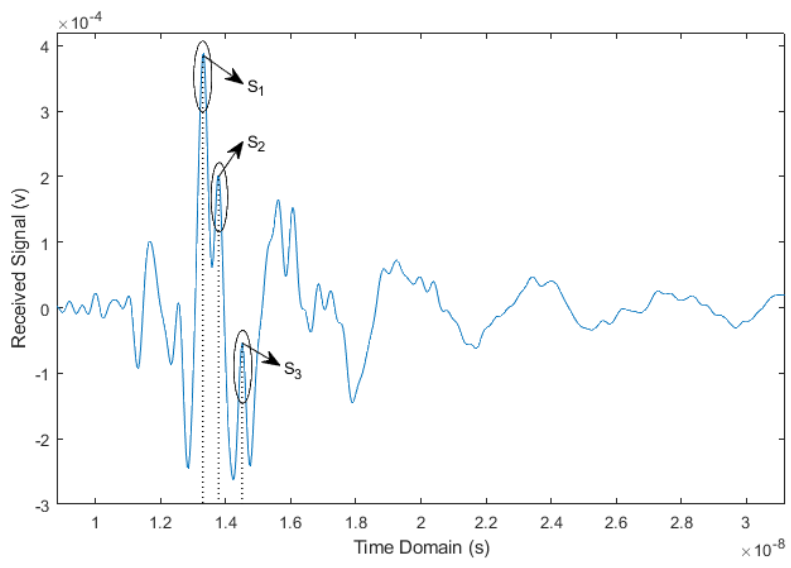

Fig. 6. A-scan of scenario A

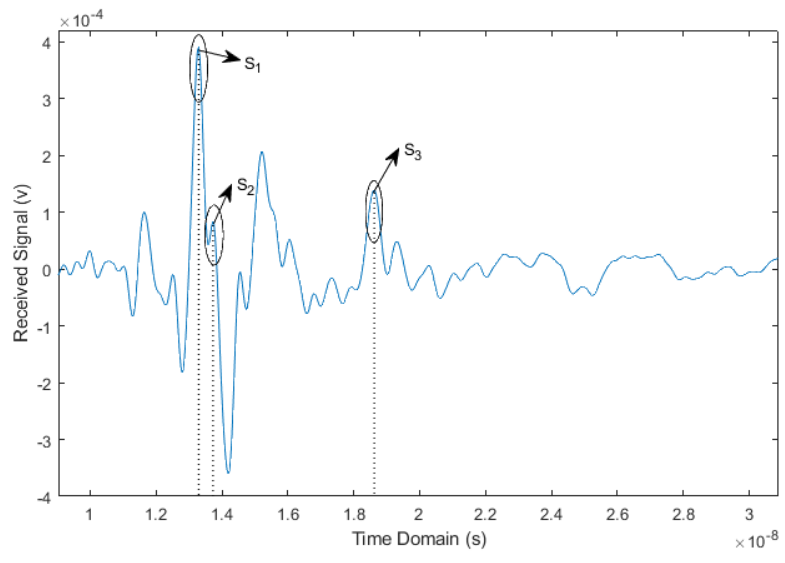

Fig. 7. A-scan of scenario B

A-scan conditions in A and B scenario, display the value of the signal received when detecting object buried in different depths. The result of taking a single point data show in sequence. Changing the sequence into time-domain needs to be done to ensure that the signal received from the objects reflection during the experiment is similar to the actual objects position. Figure 8, shows the result of the A-scan signal with S1, S2, and $\mathrm{S} 3$ peaks at $1.333 \times 10^{-8}$ second, $1.379 \times 10^{-8}$ second, and $1.4451 \times 10^{-8}$ second, respectively. The distance between the antennas and the surface 
ground is calculated by using $\Delta S_{1} S_{2}$ is $0.46 \times 10^{-9}$ second and the $\Delta S_{2} S_{3}$ is $0.72 \times 10^{-9}$ second times speed of light $(c)$ devided by two. On the other hand, in Figure 7 shows the result of the B-scan signal with $S_{1}, S_{2}$, and $S_{3}$ peaks at $1.329 \times 10^{-8}$ second, $1.376 \times 10^{-8}$ second, and $1.861 \times 10^{-8}$ second. The distance between the antennas and the surface ground is calculated by using $\Delta S_{1} S_{2}$ of $0.47 \times 10^{-9}$ second and the $\Delta S_{2} S_{3}$ of $4.85 \times 10^{-9}$ second times speed of light $(c)$ devided by two.

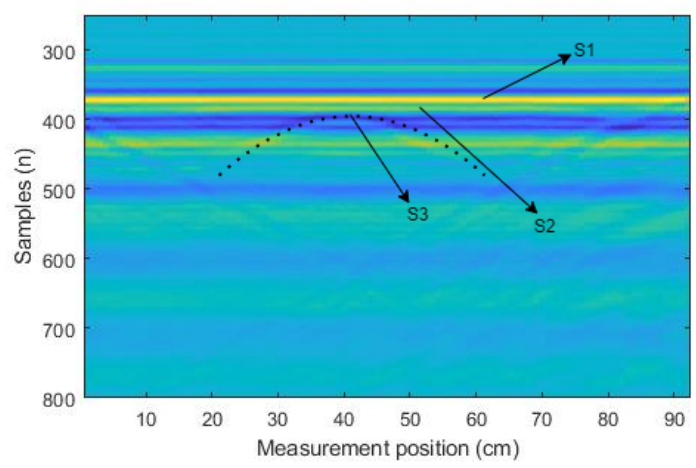

Fig. 8. B-scan of scenario A

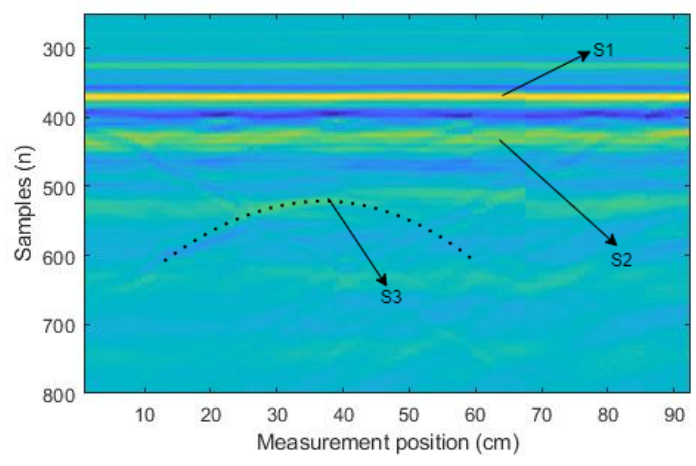

Fig. 9. B-scan of scenario B

Quantitatively, the depth of scenario A is calculated using Eq. (2) with the velocity value of $84.75 \times 10^{6} \mathrm{~m} / \mathrm{s}$ and alternating time of $2.36 \times$ $10^{-9} s$. The real depth of the antenna to the object is $10 \mathrm{~cm}$, however the estimated value for depth is $10.0005 \mathrm{~cm}$. It means the accuracy of scenario A is $99.95 \%$. On the other hand, in scenario $\mathrm{B}$, the velocity value used of $144.07 \times 10^{6} \mathrm{~m} / \mathrm{s}$ and an alternating time of $10.64 \times 10^{-9} s$. The real distance between the antenna and the object is $17 \mathrm{~cm}$, however the estimated distance of $17.00272 \mathrm{~cm}$. It means the accuracy of scenario A is $99.728 \%$. The difference in velocity produced by two scenarios is caused by heterogeneous ground.

The experiments show that the position of the object affects the interpretation and distance of

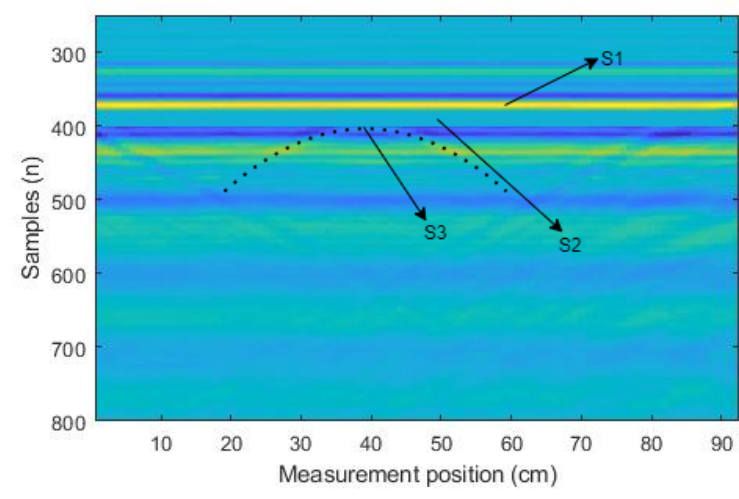

Fig. 10. scenario A without surface clutter

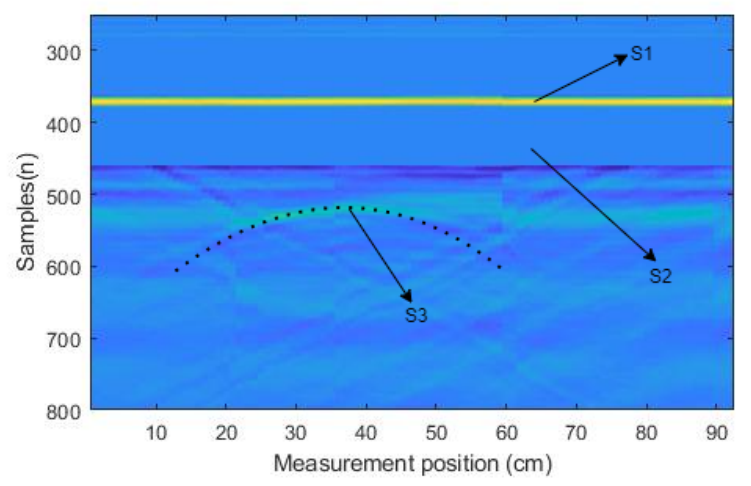

Fig. 11. scenario B without surface clutter

the reflected signal generated from the object and the surface reflection signal. In scenario $A$, the difference between object reflection and surface reflection is difficult to distinguish because the object's distance is too close, but the interpretation of the signal is clearer, as shown in Figure 8. The dotted lines on the B-scan result indicate that at the peak of the hyperbolic shape, indicating a detected object. While in scenario B, the interpretation of the signal is more subtle than scenario A, but the distance between the surface reflection signal and the object reflection signal is further apart that makes it easier to distinguish, details can be seen in Figure 9.

The process of reducing surface clutter is provided on the results of scenarios A and B by using Eq. (5). In scenario A, the reduction of surface clutter is more difficult because the distance between the surface reflection clutter and the object reflection is also reduced in the process as shown in Figure 10. While, in scenario B, surface clutter is easier to reduce because of the distance between the object's reflection and the surface reflection is wider so that the results of the hyperbolic pattern from the object's reflection are not disturbed as 


\section{Ramadhamy et al / Journal of Measurement, Electronics and Communication Systems}

shown in Figure 11.

\section{Conclusion}

This study focuses on the effect of object depth on reduce the clutter. Further depth variation is necessary to be studied to obtain more comprehensive results. At $3 \mathrm{~cm}$ depth, the process of reducing surface clutter is more difficult due to the close distance between the surface clutter and the object and not all surface clutter is reduced because it affects the hyperbolic signal of the object. In contrast, the process of reducing surface clutter at $10 \mathrm{~cm}$ depth is easier because the long distance between the surface clutter and the object.

\section{References}

[1] D. J. Daniels, "Ground Penetrating Radar, $2^{\text {nd }}$ Ed", London, United Kingdom: The Institution of Electrical Engineers, 2004.

[2] Erfansyah Ali, A. A. Pramudita and Dharu Arseno, Concrete Thickness Measurement Model for GPR, 2019 Internasional Conference on Antenna, Measurement and Application (CAMA), December 2019, pp. 125-128.

[3] Smitha N and V. Singh, Clutter reduction using background subtraction of ground penetrating radar for landmine detection, 2015 IEEE Asia Pacific Conference on Postgraduate Research in Microelectronics and Electronics (PrimeAsia), Nov 2015, pp. 1116.

[4] Alindra, Rahmayati and Wijanto, Heroe and Usman, Koredianto, "Deteksi Bentuk Objek Bawah Tanah Menggunakan Pengolahan Citra B-Scan pada Ground Penetrating Radar (GPR)," TELKA - Telekomunikasi, Elektronika, Komputasi dan Kontrol, 2017, vol. 3, pp. $73-83$.

[5] G. Nadim, Clutter reduction and detection of landmine objects in ground penetrating radar data using likelihood method, 2008 3rd International Symposium on Communications, Control and Signal Processing, March 2008, pp. 98106.

[6] Li Ting-jun, Kong Ling-jiang, and Zhou Zheng-ou, Symmetry filtering method for gpr clutter reduction, 2008 International Conference on Microwave and Millimeter Wave Technology, vol. 3, April 2008, pp. 15151517.

[7] Yano, T., Umeda, N., Hirayama, K., Baba, M., and Sakai, M., "Wave radar application to the simplified parametric roll operational guidance at actual sea," Proceedings of the 17th International Ship Stability Workshop, 10-12 June 2019, pp. 91-96.

[8] Sakai, M., Umeda, N., Yano, T., Maki, A., Yamashita, N., Matsuda A., and Terada, D., "Averaging methods for estimating parametric roll in longitudinal and oblique waves," Journal of Marine Science and Technology, vol. 23, 2018, pp. 413424.

[9] G. Zhang, L. Tsang and Y. Kuga, "Studies of the angular correlation function of scattering by random rough surfaces with and without a buried object," IEEE Transactions on Geoscience and Remote Sensing, vol. 35, no. 2, pp. 444-453, March 1997.

[10] Andini Dwi Pratiwi, D. Arseno, and A. Adya Pramudita, "Metode Identifikasi Rongga Pada Batang Kayu dengan Menggunakan Ground Penetrating Radar," 2019 e-Proceeding of Engineering, Vol.6, No.2, Agustus 2019, pp. 42384245.

[11] E. C. Utsi, "Ground Penetrating Radar Theory and Practice," United Kingdom: Joe Hayton, 2017.

[12] G. Zhang, L. Tsang and Y. Kuga, "Studies of the angular correlation function of scattering by random rough surfaces with and without a buried object," in IEEE Transactions on Geoscience and Remote Sensing, vol. 35, no. 2, pp. 444-453, March 1997.

[13] W. Xun, L. Jinbing, R. Xiaoyuan and L. Haibo, "Research on the performance evaluation method of GPR clutter suppression," IET International Radar Conference 2015, 2015, pp. 1-5.

[14] Benedetto, Andrea and Tosti, Fabio and Bianchini Ciampoli, Luca and D'Amico, Fabrizio, "An overview of ground-penetrating radar signal processing techniques for road inspections," Signal Processing, Mei 2016, vol. 132, pp. 201-209.

[15] Harry M. Jol, "Ground Penetrating Radar: Theory and Apllications," Netherlands, Elsevier Science, 2009, ISBN: 978-0-444-53348-7. 


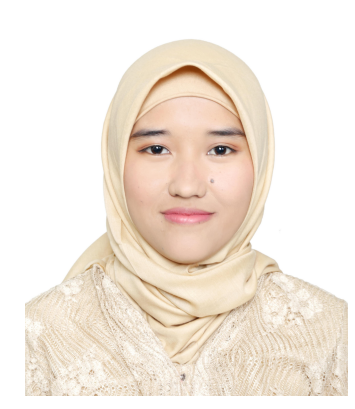

Queen Hesti Ramadhamy was born in Sungai Rumbai, in 1998. She received the bachelor's degree in telecommunication engineering from Telkom University, Indonesia in 2020. She is interested in signalling topics and therefore, she was one of the digital signal processing laboratory assistant. In addition, she also took the topic for her undergraduate thesis is about signal processing in GPR.

Erfansyah Ali, re-

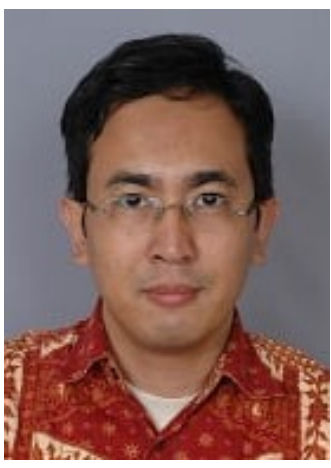
ceived his BS (2005) and MS (2012) both from Electrical Engineering Department major in Communications, Intitut Teknologi Bandung (ITB). Currently he is a lecturer at School of Electrical Engineering, Telkom University and a $\mathrm{PhD}$ student at $\mathrm{Na}$ tional Taiwan University of Science and Technology (NTUST). His research interests are in Radar Systems, RF device and Signal Processing.

Aloysius Adya Pramudita (S'08 M'12) was

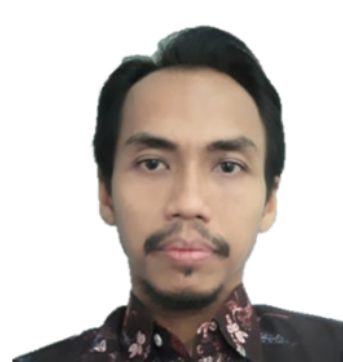
born in Klaten, Indonesia, in 1977 . He received the B.S. in electrical engineering from Gadjah Mada University, Indonesia in 2000, M.S. and Ph.D degree in electrical engineering from the Bandung Institute of Technology, Indonesia in 2005 and 2009

respectively. From 2002 to 2016, he was a Lecturer and Researcher with the Atma Jaya Catholic University, Indonesia. From 2013 to 2016, He was head of Electrical Engineering Department of Atma Jaya Univeristy and from 2016 to 2017 as head of Electrical Engineering Graduate Program of Atma Jaya Catholic University. Since 2017, he joined the Telecommunication Engineering Department of Telkom University, Bandung, Indonesia. He responsible as head of Satellite Communication and Radar Laboratory of Telkom University. He also serves as a reviewer for several technical journals and conferences in his interest area. His research interests include antenna theory and design for telecommunication and radar, electromagnetics and wave application, and radar system for contactless sensor. 